\title{
A Single Detector Versus an Array of Detectors Receiver in Free-Space Optical Communications: A Performance Comparison
}

This paper was downloaded from TechRxiv (https://www.techrxiv.org).

\section{LICENSE}

CC BY 4.0

SUBMISSION DATE / POSTED DATE

$09-01-2021 / 12-01-2021$

\section{CITATION}

Bashir, Muhammad Salman; Tsai, Ming-Cheng; Alouini, Mohamed-Slim (2021): A Single Detector Versus an Array of Detectors Receiver in Free-Space Optical Communications: A Performance Comparison. TechRxiv. Preprint. https://doi.org/10.36227/techrxiv.13550522.v1

\section{$\mathrm{DOI}$}




\title{
A Single Detector Versus an Array of Detectors Receiver in Free-Space Optical Communications: A Performance Comparison
}

Ming-Cheng Tsai, Student Member, IEEE, Muhammad Salman Bashir, Senior Member, IEEE, and Mohamed-Slim Alouini, Fellow, IEEE

\begin{abstract}
Free-space optical (FSO) communications is an important technology that will be used for supporting high data-rates in the backhaul of next generation of wireless communication networks. In this paper, we have compared the probability of error performance of two types of receivers used in FSO today: a receiver based on a single detector, and a receiver based on an array of detectors. The performance of these two receivers is compared for a number of fusion algorithms for an array of detectors such as equal gain combiner (EGC), selection combiner (SC), switched combiner (SWC) and the maximal ratio combiner (MRC). From this study, we conclude that even though the array of detectors adds more noise in the sufficient statistic by virtue of large number of detectors, using more computationally expensive fusion algorithms (such as SC and MRC) can help us achieve a superior probability of error performance as opposed to a single-detector receiver for practical channel conditions.
\end{abstract}

\section{Index Terms}

Array of detectors, equal gain combiner, free-space optics, maximal ratio combiner, probability of error, selection combiner, single-detector, switched combiner.

M. -C. Tsai, M. S. Bashir and M. -S. Alouini are with the King Abdullah University of Science and Technology (KAUST), Thuwal 239556900, Kingdom of Saudi Arabia. e-mail: (mingcheng.tsai@kaust.edu.sa, salman.bashir@alumni.purdue.edu, slim.alouini@kaust.edu.sa). 


\section{INTRODUCTION}

Free-space optics is slated to become an enabler of high data-rates in the backhaul of next generation of wireless communication networks due to the availability of large chunks of unused spectrum in the optical domain of the electromagnetic spectrum [1]. However, due to the narrow beamwidth associated with the optical signal (the beamwdith angle is proportional to $\frac{\lambda}{D}$ where $\lambda$ is the wavelength of light and $D$ is diameter of transmit aperture), pointing and tracking requirements become more stringent for the performance of an FSO communications link. Thus, the pointing and tracking subsystems form an important part of an FSO communication system. At the receiver, the received light is focused on a detection element - which is either a single-detector or an array of detectors - that lies in the focal plane of the aperture lens. Typically, noncoherent energy detection (intensity modulation/direct detection or IM/DD) scheme is used in FSO receivers because of its relative simplicity as opposed to coherent receivers.

Typically, IM/DD based detector arrays are used in deep-space optical communications where the link distances are so long that the transmitted signal pulse is decoded based on a few received signal photons [2], [3]. Another reason for a low number of received signal photons is the phenomenon of "blocking" associated with avalanche photodetectors (APD) that are operated in Geiger mode as photon counters. The blocking occurs because the detection of the first photon causes an avalanche of electrons due to high gain of an APD, and this avalanche has to be quenched by an avalanche recovery circuit, and the bias has to be restored, before the next incoming signal photon can be detected. Thus, the APD gets "blocked" for a few microseconds after a single photon detection before it is ready to detect the next incoming photon. Thus, for a deep space optical communication receiver, it makes sense to spread the beam energy over an array of individual detectors in order to minimize the effect of blocking, and thereby increase the number of detected photons per pulse to improve the SNR.

A single-detector IM/DD receiver based on a single PIN photodiode is typically used for short link terrestrial FSO systems where the received signal power is enough for the purpose of a satisfactory bit error rate performance. The PIN diodes are not as sensitive as an APD when it comes to the detection of low-energy pulses. However, they do a satisfactory job for moderate to high amounts of received signal power. For a more detailed comparison on these two types of detectors, a good reference is [4].

Fig. 1 shows the profile of the Gaussian-approximated airy pattern in the focal plane, whereas Fig. 2 depicts an array of detectors and a single-detector receiver. 


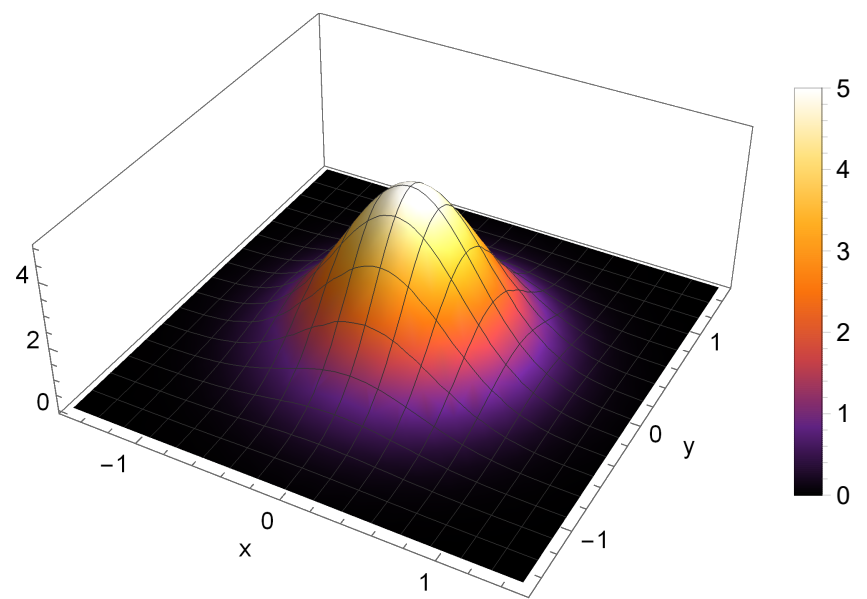

Fig. 1. Profile of the incident beam in the focal plane.
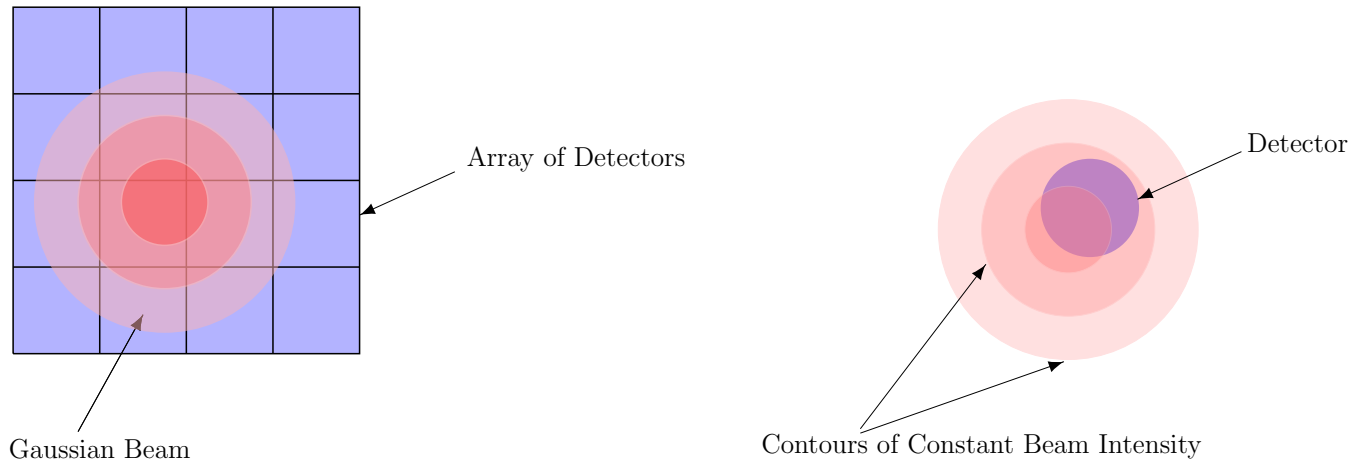

Fig. 2. This figure shows an array of detectors (on the left) versus a single-detector receiver (right).

\section{A. Motivation}

One might ask the following question: "Should one choose a single PIN detector, or should one choose an array of PIN detectors for short-to-medium link FSO communications ${ }^{1}$ in order to minimize the probability of error?" The answer to this question depends on a number of factors. So, before we answer this question, it is important that we first understand the pros and cons of an array of detectors versus a single-detector receiver.

\section{1) Pros of a Single Detector Versus an Array of Detectors}

Let us initially look at some of the pros of a single-detector receiver as opposed to an array of detectors. Firstly, the bandwidth of the detector depends on its physical size or surface area [5]. A larger surface area leads to a larger junction capacitance which implies a slower response to the changing optical signal. Thus, in order to improve the switched response of the detector, it is important to reduce its size so that the detector can detect fast changes in the received optical signal. Additionally, a small detector also adds 


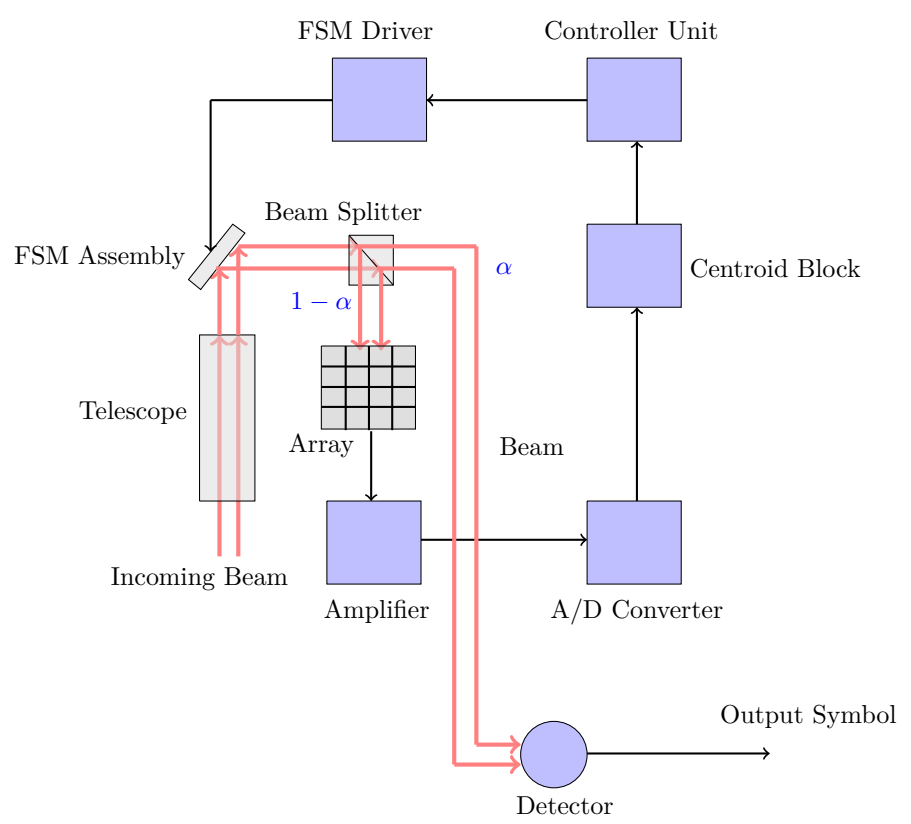

Fig. 3. This figure shows the block diagram of a single-detector receiver. Only a fraction of received energy (denoted by $\alpha$ ) goes into the data channel, and $(1-\alpha)$ finds its way into the tracking channel. The parameter $\alpha$ can be varied between 0 and 1 by turning a knob on a variable beam splitter.

less noise in the sufficient statistic (the amount of dark current and background radiation captured by a detector is proportional to its area). For an array of detectors, the number of detectors $M \triangleq \frac{\mathcal{A}}{A}$ where $\mathcal{A}$ is the area of the detector array, and $A$ is the area per detector. The area of the array $\mathcal{A}$ depends on the variance of the pointing error (also called jitter in the FSO literature) or the deviation of the beam about its mean position. The magnitude of the pointing error variance depends on the physical parameters of the channel: The larger the variance of the pointing error, the larger the area $\mathcal{A}$ required in order to effectively capture all the beam energy. The area of each detector $A$ has to be small enough in order to support a high enough data rate of symbol transmission on the channel. Thus, it may be that a large variance of the jitter or beam deviation may lead to a large number of detectors $M$ in the array which may be too complex-both from the hardware as well as the computational resource perspectives-to realize.

Secondly, an array of detectors will also add more noise in the sufficient statistic since each detector in the array will be adding background radiation/thermal noise into the total noise power. In order to counter this noise resulting from each detector, a fusion algorithm—such as a maximal ratio combiner or a selection combiner-may be used that will weight the output of each detector optimally or suboptimally before the fusion takes place. However, the inclusion of such a fusion device will add its own computational complexity to the resulting receiver. 


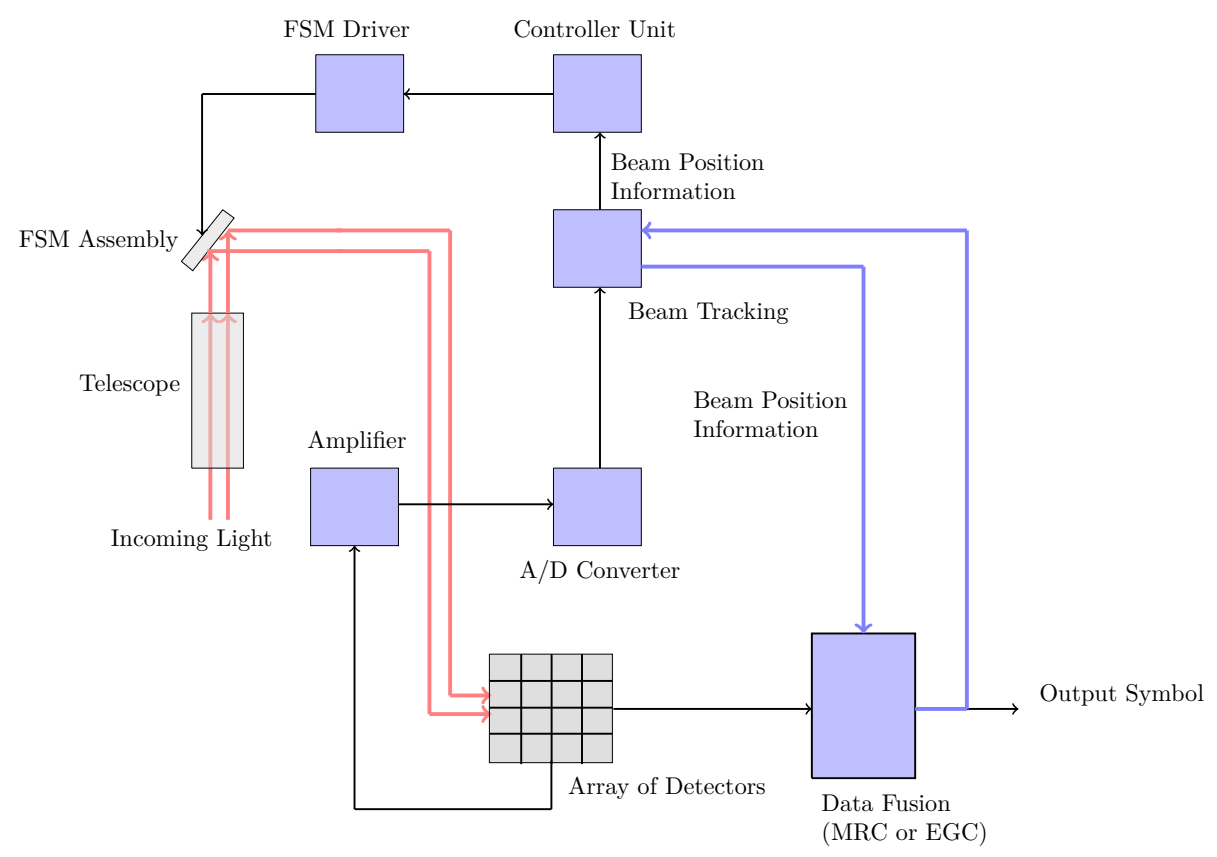

Fig. 4. This figure shows the block diagram of a detector array receiver.

\section{2) Pros of an Array of Detectors Versus a Single Detector}

Now, we look at some of the pros of a detector array versus a single-detector receiver. Firstly, because of angle-of-arrival (AoA) fluctuations of the beam at the receiver, the focused light beam impinging on a (small) single detector is always moving about or deviating about its mean position. We term this random deviation as the pointing error or jitter in this paper. Due to the pointing error, the beam radius has to be kept large enough so that any deviations of the beam do not lead to an outage of the signal at the detector. Additionally, even if the center of the detector and the center of the beam are aligned, the detector does not capture all the energy in the beam. If we replace this single detector with an array of detectors, and make the area of the array large enough by adding enough number of detectors, we may be able to capture all the received signal energy after the aperture lens.

Secondly, for a single-detector receiver, a separate beam tracking assembly/channel is needed. The tracking assembly is typically made up of a quad-detector or a $2 \times 2$ detector array where each detector has an area large enough so as to accommodate for any deviation of the beam or beam wander. Thus, the net fraction of energy going into the data channel (indicated by $\alpha$ in Fig. 3) is reduced in order to divert a fraction of received energy into the tracking channel. For the array of detectors, a separate beam tracking assembly is not required since the same array can be used for beam tracking as well as symbol detection (please see Fig. 4). 
Thus, in order to answer the question posed at the beginning of Section I-A, we have to make a performance comparison (bitter error rate and computational complexity comparison) between the singledetector and the detector array receivers while keeping in mind the various factors and conditions that are discussed in the pros and cons sections (Section I-A2 and Section I-A1). This comparison will highlight the advantages (or disadvantages) of one scheme over another, and will give the reader an idea of which system to choose under a given set of system parameters and conditions. This is the main motivation behind the study conducted in this paper.

\section{Literature Review and Model Assumptions}

\section{A. Background Literature Review}

The authors in [2], [6] have proposed beam position estimation and tracking algorithms for a detector array photon-counting receiver concerning a joint beam position tracking and symbol detection scheme for PPM symbols. The authors in [7] analyze the probability of error in detail for the joint beam position tracking and symbol detection system, and show that the probability of error of a maximal ratio combining scheme is minimized if the beam is tracked accurately enough in time. The authors in [8] consider time synchronization schemes for a detector array receiver in a photon-counting channel in deep space optical communications. The authors in [9] have considered the communications performance for an array of detectors and discussed the effects of the number of detectors and the beam parameter information on the performance. They showed that for a maximal ratio combining scheme, the probability of error decays monotonically with the number of detectors in the array if the area of the array is kept fixed. The authors in [10] have deliberated upon the signal acquisition performance obtained with an array of detectors receiver for a photon-counting optical channel. The work [11] analyzes the performance of a free-space optical multiple-input single-output receiver for noncoherent and coherent beam combining schemes and pointing errors. For more information on the performance of an array of detectors in a deep-space optical communications, references [12], [13], [14] provide an excellent read.

Regarding pointing errors, the authors in [15] consider the optimization of outage capacity of an FSO link in terms of the beam radius. The link employs a single detector at the receiver, and the outage capacity is optimized for a Gaussian beam. The authors in [16] examine the maximization of link availability as a function of beam radius for a single-detector FSO receiver.

Finally, references [17], [18], [19] present a good overview of diversity combining schemes in wireless communications. 


\section{B. Contributions of This Paper}

The references mentioned in the literature review section either focus only on the performance analysis of an array of detectors, or they solely deal with the performance evaluation of single-detector receiver without considering a comparison of the two systems. In our opinion, there is no reference available in FSO literature that has attempted to compare the performance of these two systems under different channel conditions.

In this paper, we have compared the bit error rate performance of a single-detector receiver with a detector array receiver for a pulse position modulation (PPM) scheme. In this regard, we have compared the performance keeping in view a number of physical factors such as the pointing error or jitter variance $\sigma_{R}^{2}$, the beam or spot radius $\rho$ in the focal plane, and the SNR. Additionally, concerning the array of detectors, the probability of error performance for different fusion algorithms such as equal gain combiner (EGC), selection combiner (SC), switched combiner (SWC), and the maximal ratio combiner (MRC) are compared.

\section{Model Assumptions}

Without a loss of generality, we make the following assumptions before we begin the analysis in this study:

1) The shape of the airy pattern or the spot on the focal plane is approximated by a Gaussian distribution in two dimensions. Additionally, we assume that the noise intensity is distributed uniformly over the array. The noise results due to thermal effect that leads to dark current in detectors. We assume that the effect of unwanted background radiation - that is location dependent on the array instead of being uniformly distributed-is minimized significantly by the use of optical filters, and hence the background radiation effect is negligible.

2) The Gaussian beam center $\left(x_{0}, y_{0}\right)$ deviates about the origin $(0,0)$ in the focal plane. The origin $(0,0)$ is also the location of the center of the detector in the single-detector receiver, or in case of the array of detectors, $(0,0)$ is the center of the array. The beam moves about in a random fashion due to vibrations of the transmitter/receiver mechanical assemblies and the beam wander due to atmospheric turbulence. The deviation of the center of the beam from the origin is represented by random variable $R$. The random variable $R$ is also known as the pointing error in our case. 
3) The distribution of the pointing error $R$ is assumed to be Rayleigh. In future work, we will consider a more general model such as a Beckmann distribution [16].

4) The detector radius $a$ for the single-detector receiver is smaller than the radius $\rho$ of the beam. A circular detector is chosen in this case.

5) The shape of the array is a square and the size/area of the array depends on the standard deviation of pointing error $\sigma_{R}$. Additionally, the shape of each detector in the array is also a square so that all the detectors fill up the space inside the array. Thus, the optical fill factor of a hundred percent is assumed in this case ${ }^{2}$.

\section{Organization of This Paper}

This paper is organized as follows. Section III discusses the signal and noise model and the probability of error expressions for the single-detector receiver. Section III-A considers the probability of error for a single detector case as a function of the area of the detector for the case of perfect alignment (or zero pointing error). Section IV considers the probability of error for an array of detectors, and the probability of error expressions for different fusion algorithms are either presented or derived. Section V contains the commentary on simulation results and Section VI argues a brief complexity analysis between the single-detector and the detector array receivers. Section VII sums up the conclusions of our study.

Fig. 5 shows the list of important mathematical symbols used in this paper.

\begin{tabular}{|c|c|}
\hline Notation & Name \& Units \\
\hline$\rho$ & Beam radius (mm) \\
\hline$\left(x_{0}, y_{0}\right)$ & Beam center location \\
\hline$\lambda_{p}$ & Peak signal intensity (Watt) \\
\hline$a$ & Radius of single detector (mm) \\
\hline$\alpha$ & Power split factor of single detector \\
\hline$\sigma_{R}^{2}$ & Jitter or pointing error variance (mm) \\
\hline$N_{0}$ & Noise Parameter (Watts/mm $\left.{ }^{2}\right)$ \\
\hline$M$ & Number of detectors in the array \\
\hline $\mathcal{A} \mid$ & Area of detector array (mm ${ }^{2}$ ) \\
\hline $\mathcal{M}$ & PPM modulation order \\
\hline$\Lambda_{m}$ & Signal intensity in $m$ th detector of the array \\
\hline
\end{tabular}

Fig. 5. List of Mathematical Symbols

${ }^{2}$ Even if the optical fill factor is less than a hundred percent, we can get close to a hundred percent effect by using a microlens array before the array of detectors in the focal plane. 


\section{Probability of Error for a Single-Detector Receiver}

Let the intensity profile of the airy pattern at the focal plane be modeled by a Gaussian distribution. Then, for any point $(x, y)$ in the focal plane, the total intensity is given by

$$
\lambda_{s}(x, y) \triangleq \frac{\lambda_{p}}{\rho^{2}} \exp \left(\frac{-\left(x-x_{0}\right)^{2}-\left(y-y_{0}\right)^{2}}{2 \rho^{2}}\right)+N_{0}
$$

where $\lambda_{p}$ is peak intensity in Watts $/ \mathrm{mm}^{2}, \rho$ is the beam radius in millimeters, $\left(x_{0}, y_{0}\right)$ is the beam center location, and $N_{0}$ is the constant noise intensity or power in Watts $/ \mathrm{mm}^{2}$.

Let the total received signal energy be given by

$$
P_{R}=\int_{-\infty}^{\infty} \int_{-\infty}^{\infty} \frac{\lambda_{p}}{2 \pi \rho^{2}} \exp \left(-\frac{\left(x^{2}+y^{2}\right)}{2 \rho^{2}}\right) d y d x
$$

Then the signal energy received by a detector whose center lies at a distance $R$ from the center of the beam is,

$$
P(R)=\int_{R-a}^{R+a} \int_{-\sqrt{a^{2}-(x-R)^{2}}}^{+\sqrt{a^{2}-(x-R)^{2}}} \alpha \frac{\lambda_{p}}{2 \pi \rho^{2}} \exp \left(-\frac{\left(x^{2}+y^{2}\right)}{2 \rho^{2}}\right) d y d x
$$

where $0<\alpha<1$ is the power split factor. For the single-detector receiver, the fraction $\alpha$ of the total received power goes into the data channel assembly, and the fraction $(1-\alpha)$ goes into the tracking assembly (please see Fig. 3). As discussed before, the deviation $R$ is modeled by a Rayleigh distribution whose scale parameter is denoted by $\sigma_{R}$.

For the purpose of computing the probability of error, let the output of the detector be given by

$$
Y=S(R)+V
$$

where

$$
S(R)=\int_{0}^{T_{s}} P(R) d t
$$

and $V \sim \mathcal{N}\left(0, \sigma_{v}^{2}\right)$ is the noise due to background radiation and thermal effect. The quantity $\sigma_{v}^{2}$ represents the variance of noise due to background radiation, and it is defined as $\mu_{v}=N_{0} \pi a^{2}$, where $N_{0}$ is a constant measured in in Watts $/ \mathrm{mm}^{2}$. Let us transmit symbol $0 \leq j<\mathcal{M}$ for an $\mathcal{M}$-PPM scheme. For an EGC receiver that operates on the maximum likelihood principle, we say that a symbol $j$ is detected if $Y_{j}>Y_{i}$ 
for all $i \neq j, 0 \leq i<\mathcal{M}$. Then, the conditional probability of the correct decision is [7]

$$
\mathbb{P}(\mathcal{C} \mid j)=\left(P\left(\left\{Y_{j}-Y_{i}>0\right\}\right)\right)^{\mathcal{M}-1},
$$

where $\mathcal{C}$ represents the event that a correct decision is made on a PPM symbol, $Y_{j} \sim \mathcal{N}\left(S(R), \sigma_{v}^{2}\right)$ and $Y_{i} \sim \mathcal{N}\left(0, \sigma_{v}^{2}\right)$. For an equiprobable symbol scheme, we have that $\mathbb{P}(\mathcal{C} \mid j)=\mathbb{P}(\mathcal{C})$. Therefore, the probability of error, $\mathbb{P}(\mathcal{E})$, is $\mathbb{P}(\mathcal{E})=1-\mathbb{P}(\mathcal{C})$.

The quantity $\left(Y_{j}-Y_{i}\right) \sim \mathcal{N}\left(S(R), 2 \sigma_{v}^{2}\right)$. Therefore,

$$
\mathbb{P}(\mathcal{E} \mid R=r)=1-\left(Q\left(-\frac{S(r)}{\sqrt{2} \sigma_{v}}\right)\right)^{\mathcal{M}-1}
$$

and

$$
\mathbb{P}(\mathcal{E})=\int_{0}^{\infty} P(\mathcal{E} \mid r) f_{R}(r) d r=\int_{0}^{\infty}\left(1-\left(Q\left(-\frac{S(r)}{\sqrt{2} \sigma_{v}}\right)\right)^{\mathcal{M}-1}\right) f_{R}(r) d r
$$

The distribution of $R$ is given by

$$
f_{R}(r)=\frac{r}{\sigma^{2}} \exp \left(-\frac{r^{2}}{2 \sigma^{2}}\right), \quad r>0 .
$$

Additionally, for a high data-rate scheme (very small symbol period $T_{s}$ ), $S(R) \approx P(R) T_{s}$. For the sake of simplicity, let $T_{s}$ be equal to one time unit. Then,

$$
\mathbb{P}(\mathcal{E})=\int_{0}^{\infty}\left(1-\left(Q\left(-\frac{P(r)}{\sqrt{2} \sigma_{v}}\right)\right)^{\mathcal{M}-1}\right) \frac{r}{\sigma_{R}^{2}} \exp \left(-\frac{r^{2}}{2 \sigma_{R}^{2}}\right) d r
$$

The $\mathbb{P}(\mathcal{E})$ for the single detector case is minimized in terms of the beam radius $\rho$ :

$$
\begin{array}{ll}
\underset{\rho}{\operatorname{minimize}} & P(\mathcal{E}) \\
\text { subject to } & \rho_{0}<\rho<\rho_{1},
\end{array}
$$

where $\rho_{0}, \rho_{1} \in \mathbb{R}$. We denote the minimized $\mathbb{P}(\mathcal{E})$ by $\mathbb{P}(\mathcal{E})^{*}$ and the optimum $\rho$ by $\rho^{*}$.

\section{A. Probability of Error for the Limiting Case of Detector Radius and Zero Jitter}

In this section, we digress to show the effect of detector radius $a$ on the probability of error of a single-detector receiver under the ideal condition that the pointing error is zero. We will examine the error probability for two asymptotic regimes: when $a \rightarrow 0$ and when $a \rightarrow \infty$. Especially, the effect of 
small $a$ on the probability of error may not be straightforward to predict since the signal and the noise power both go to zero as $a \rightarrow 0$.

The signal energy captured by the single detector-when its center is perfectly aligned with the beam center-is

$$
P(0)=\int_{0}^{a} \alpha \lambda_{p} \frac{r}{\rho^{2}} \exp \left(-\frac{r^{2}}{2 \rho^{2}}\right) d r=\alpha \lambda_{p}\left(1-\exp \left(-\frac{a^{2}}{2 \rho^{2}}\right)\right) .
$$

The probability of error from (10) is

$$
\mathbb{P}(\mathcal{E})=1-\left(Q\left(-\frac{P(0)}{\sqrt{2} \sigma_{v}}\right)\right)^{\mathcal{M}-1}
$$

where $\sigma_{v}^{2} \triangleq N_{0} \pi a^{2}$. Thus,

$$
\mathbb{P}(\mathcal{E})=1-\left(Q\left(-\frac{\alpha \lambda_{p}\left(1-\exp \left(-\frac{a^{2}}{2 \rho^{2}}\right)\right)}{\sqrt{2 N_{0} \pi a^{2}}}\right)\right)^{\mathcal{M}-1}
$$

When $a \rightarrow 0$, we have that the numerator and the denominator of the argument of $Q$ both go to zero. In this case, we apply the L'Hôpital's Rule to the argument to obtain

$$
\begin{aligned}
& \lim _{a \rightarrow 0} \frac{\alpha \lambda_{p}\left(1-\exp \left(-\frac{a^{2}}{2 \rho^{2}}\right)\right)}{\sqrt{2 N_{0} \pi a^{2}}}=\lim _{a \rightarrow 0} \frac{\frac{d}{d a} \alpha \lambda_{p}\left(1-\exp \left(-\frac{a^{2}}{2 \rho^{2}}\right)\right)}{\frac{d}{d a} \sqrt{2 N_{0} \pi a^{2}}} \\
& =\lim _{a \rightarrow 0} \frac{\alpha / \rho^{2} \lambda_{p} \exp \left(-\frac{a^{2}}{2 \rho^{2}}\right) \sqrt{2 N_{0} \pi a^{2}}}{2 N_{0} \pi}=0 .
\end{aligned}
$$

Since $Q(0)=\frac{1}{2}$, we have that,

$$
\lim _{a \rightarrow 0} \mathbb{P}(\mathcal{E})=1-\left(\frac{1}{2}\right)^{\mathcal{M}-1}
$$

When $a \rightarrow \infty$, we have that the argument of the $Q$ function in (15) is

$$
\lim _{a \rightarrow \infty} \frac{\alpha \lambda_{p}\left(1-\exp \left(-\frac{a^{2}}{2 \rho^{2}}\right)\right)}{\sqrt{2 N_{0} \pi a^{2}}}=0
$$

which implies that $\lim _{a \rightarrow 0} \mathbb{P}(\mathcal{E})=\lim _{a \rightarrow \infty} \mathbb{P}(\mathcal{E})=1-\left(\frac{1}{2}\right)^{\mathcal{M}-1}$. This gives us a hint that for a fixed beam radius $\rho$, there might be an optimum detector radius (or radii) $a^{*}$ that will minimize the probability of error.

Fig. 6 shows the probability of error (see (15)) as a function of detector radius $a$ for different values of beam radius $\rho$. We note that when $a \rightarrow 0$, the probability of error takes a hit since the rate of decrease in 
the signal energy captured by the detector is greater than the rate of decrease in the total noise power. On the other hand, as $a \rightarrow \infty$, the captured signal power approaches $\lambda_{p}$, but the noise power goes to infinity. Hence, the probability of error degrades significantly-approaches $1-\left(\frac{1}{2}\right)^{\mathcal{M}-1}$ - in this case.

Finally, even though we assume that the pointing error is zero for the sake of simplicity in this short analysis, the results obtained here can be easily extended to the case when the pointing error is not zero.

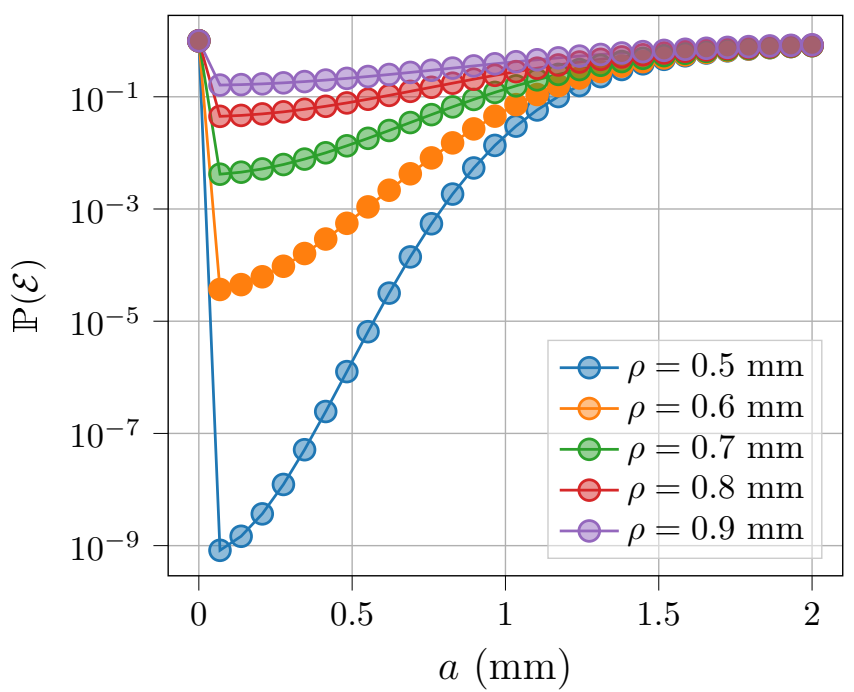

Fig. 6. This figure shows the probability of error as a function of detector radius $a$ for different values of beam radius $\rho$. The value of peak signal power $\lambda_{p}=1$ Watts, and $N_{0}=0.05$ Watts $/ \mathrm{mm}^{2}$. These curves are plotted for an 8-PPM scheme.

\section{Probability of Error of an Array of Detectors}

\section{A. Equal Gain Combiner}

It can be shown that the probability of error obtained with an array of detectors for an equal gain combining scheme is given by [11]

$$
\mathbb{P}\left(\mathcal{E} \mid x_{0}, y_{0}\right)=\mathbb{P}(\mathcal{E})=1-\left(Q\left(-\frac{\sum_{m=1}^{M} \Lambda_{m}}{\sqrt{2 N_{0} \mathcal{A}}}\right)\right)^{\mathcal{M}-1}
$$

where $\Lambda_{m}$ is the signal power in the $m$ th detector of the array: $\Lambda_{m} \triangleq \iint_{A_{m}} \frac{\lambda_{p}}{2 \pi \rho^{2}} \exp \left(-\frac{\left(x-x_{0}\right)^{2}+\left(y-y_{0}\right)^{2}}{2 \rho^{2}}\right) d y d x$. 


\section{B. Maximal Ratio Combiner}

It can be shown that the probability of error for the maximal ratio combiner is [11]

$$
\mathbb{P}\left(\mathcal{E} \mid x_{0}, y_{0}\right)=1-\left(Q\left(-\sqrt{\frac{\sum_{m=1}^{M} \Lambda_{m}^{2}}{2 N_{0} A^{(M)}}}\right)\right)^{\mathcal{M}-1},
$$

where $A^{(M)}$ is the area of a single detector in the array and it is a function of $M$ if $\mathcal{A}$ is fixed.

\section{Selection Combiner}

In this section, we look at two types of selection combining schemes: the SC (Max SNR) and the SC (Max Output).

1) $S C(\operatorname{Max} S N R)$

In this case, we choose the detector that has the highest signal power (or SNR since noise power in each detector is the same). For this scenario, we note that the information of beam center location $\left(x_{0}, y_{0}\right)$ is required in order to select the detector with the maximum SNR. For SC (Max SNR), we choose a detector of the array whose signal power is

$$
\Lambda_{\max } \triangleq \max \left(\Lambda_{1}, \Lambda_{2}, \ldots, \Lambda_{M}\right)
$$

The output of the detector containing the maximum SNR is denoted by $Y_{s}$ during a PPM signal slot, and the output of the same detector during a noise-only slot is denoted by $Y_{n}$. For $\mathcal{M}$-PPM, the probability of a correct decision in this case is

$$
\mathbb{P}\left(\mathcal{C} \mid x_{0}, y_{0}\right)=\left(\mathbb{P}\left(\left\{Y_{s}>Y_{n}\right\}\right)\right)^{\mathcal{M}-1}
$$

We know that $Y_{n} \sim \mathcal{N}\left(0, N_{0} A^{(M)}\right)$. Then $P(\mathcal{C})=\left(F_{Y_{n}}\left(Y_{s}\right)\right)^{\mathcal{M}-1}$ which implies that the conditional probability is

$$
\mathbb{P}\left(\left\{Y_{n}<Y_{s}\right\} \mid\left\{Y_{s}=y\right\}\right)=F_{Y_{n}}(y)=Q\left(-\frac{y}{\sqrt{N_{0} A^{(M)}}}\right)
$$

Therefore,

$$
\mathbb{P}\left(\left\{Y_{n}<Y_{s}\right\}\right)=\int_{-\infty}^{\infty} Q\left(-\frac{y}{\sqrt{N_{0} A^{(M)}}}\right) f_{Y_{s}}(y) d y
$$


where

$$
f_{Y_{s}}(y)=\frac{1}{\sqrt{2 \pi N_{0} A^{(M)}}} \exp \left(-\frac{\left(y-\Lambda_{\max }\right)^{2}}{2 N_{0} A^{(M)}}\right)
$$

Thus,

$$
\mathbb{P}\left(\mathcal{E} \mid x_{0}, y_{0}\right)=1-\mathbb{P}\left(\mathcal{C} \mid x_{0}, y_{0}\right)=1-\left(\int_{-\infty}^{\infty} Q\left(-\frac{y}{\sqrt{N_{0} A^{(M)}}}\right) f_{Y_{s}}(y) d y\right)^{\mathcal{M}-1}
$$

a) Asymptotic Result: $M \rightarrow \infty$ : For large $M$, the signal energy in the detector having maximum SNR (for any $\left.\left(x_{0}, y_{0}\right)\right)$ is approximately

$$
S \approx \frac{\lambda_{p}}{2 \pi \rho^{2}} A^{(M)}
$$

Thus, in this case, the probability of error expression concering an $\mathcal{M}$-PPM for large $M$ is

$$
\mathbb{P}\left(\mathcal{E} \mid x_{0}, y_{0}\right) \approx 1-\left(Q\left(-\frac{\frac{\lambda_{p}}{2 \pi \rho^{2}} A^{(M)}}{\sqrt{2 N_{0} A^{(M)}}}\right)\right)^{\mathcal{M}-1}=1-\left(Q\left(-\frac{\frac{\lambda_{p}}{2 \pi \rho^{2}} \sqrt{A^{(M)}}}{\sqrt{2 N_{0}}}\right)\right)^{\mathcal{M}-1}
$$

Since $M \rightarrow \infty \Longrightarrow A^{(M)} \rightarrow 0$, for (28) we have that

$$
\lim _{M \rightarrow \infty} \mathbb{P}\left(\mathcal{E} \mid x_{0}, y_{0}\right)=1-\left(\frac{1}{2}\right)^{\mathcal{M}-1}
$$

which is the same asymptotic expression as the probability of error for the single detector case (please see (17)).

\section{2) SC (Max Output)}

In this case, we choose the detector that has the largest output for the purpose of detecting the symbol. For the signal slot of a PPM symbol:

$$
Y_{\max }^{(s)} \triangleq \max \left(Y_{1}^{(s)}, Y_{2}^{(s)}, \ldots, Y_{M}^{(s)}\right)
$$

where $Y_{m}^{(s)} \sim \mathcal{N}\left(\Lambda_{m}, N_{0} A^{(M)}\right)$. Similar to this argument, we have that when we have a noise-only slot,

$$
Y_{\max }^{(n)} \triangleq \max \left(Y_{1}^{(n)}, Y_{2}^{(n)}, \ldots, Y_{M}^{(n)}\right)
$$

where $Y_{m}^{(n)} \sim \mathcal{N}\left(0, N_{0} A^{(M)}\right)$. Now,

$$
\mathbb{P}(\mathcal{C})=\left(\mathbb{P}\left(\left\{Y_{\max }^{(s)}>Y_{\max }^{(n)}\right\}\right)\right)^{\mathcal{M}-1}
$$


In this case,

$$
\begin{aligned}
\mathbb{P}\left(\left\{Y_{\max }^{(n)}<Y_{\max }^{(s)}\right\} \mid\left\{Y_{\max }^{(s)}=y\right\}\right) & =\mathbb{P}\left(\left\{Y_{\max }^{(n)}<y\right\}\right)=\mathbb{P}\left(\left\{Y_{1}^{(n)}<y\right\} \cap\left\{Y_{2}^{(n)}<y\right\} \cap \ldots \cap\left\{Y_{M}^{(n)}<y\right\}\right) \\
& =\left(Q\left(-\frac{y}{\sqrt{N_{0} A^{(M)}}}\right)\right)^{M} .
\end{aligned}
$$

Now, the cumulative distribution function of $Y_{\max }^{(s)}$ is

$$
\begin{aligned}
F_{Y_{\max }}(y) & \triangleq \mathbb{P}\left(\left\{Y_{\max }^{(s)}<y\right\}\right)=\mathbb{P}\left(\left\{Y_{1}^{(s)}<y\right\} \cap\left\{Y_{2}^{(s)}<y\right\} \cap \ldots \cap\left\{Y_{M}^{(s)}<y\right\}\right) \\
& =\prod_{m=1}^{M} Q\left(-\frac{\left(y-\Lambda_{m}\right)}{\sqrt{N_{0} A^{(M)}}}\right) .
\end{aligned}
$$

Since $\frac{\partial}{\partial x} Q(x)=-\frac{1}{\sqrt{2 \pi}} \exp \left(-\frac{x^{2}}{2}\right)$, we have that the probability density function of $Y_{\max }^{(s)}$ is

$$
\begin{aligned}
f_{Y_{\max }}(y) & =\sum_{m=1}^{M}\left(\frac{\partial}{\partial y} Q\left(-\frac{\left(y-\Lambda_{m}\right)}{\sqrt{N_{0} A^{(M)}}}\right) \prod_{\substack{i=1 \\
i \neq m}}^{M} Q\left(-\frac{\left(y-\Lambda_{i}\right)}{\sqrt{N_{0} A^{(M)}}}\right)\right) \\
& =\sum_{m=1}^{M}\left(\frac{1}{\sqrt{2 \pi N_{0} A^{(M)}}} \exp \left(-\frac{\left(y-\Lambda_{m}\right)^{2}}{2 N_{0} A^{(M)}}\right) \prod_{\substack{i=1 \\
i \neq m}}^{M} Q\left(-\frac{\left(y-\Lambda_{i}\right)}{\sqrt{N_{0} A^{(M)}}}\right)\right) .
\end{aligned}
$$

Therefore,

$$
\mathbb{P}\left(\left\{Y_{\max }^{(n)}<Y_{\max }^{(s)}\right\}\right)=\int_{-\infty}^{\infty}\left(Q\left(-\frac{y}{\sqrt{N_{0} A^{(M)}}}\right)\right)^{M} f_{Y_{\max }}(y) d y
$$

where $f_{Y_{\max }}(y)$ is given by (36). Finally, the probability of error is

$$
\mathbb{P}\left(\mathcal{E} \mid x_{0}, y_{0}\right)=1-\mathbb{P}\left(\mathcal{C} \mid x_{0}, y_{0}\right)=1-\left(\int_{-\infty}^{\infty}\left(Q\left(-\frac{y}{\sqrt{N_{0} A^{(M)}}}\right)\right)^{M} f_{Y_{\max }}(y) d y\right)^{\mathcal{M}-1}
$$

a) Asymptotic Result: $M \rightarrow \infty$ : For the noise-only slot of $\mathcal{M}$-PPM, we know (from (33)) that the maximum output — denoted by $Y_{\max }^{(n)}$ - has the cumulative distribution given by $F(y)=\left(Q\left(-\frac{y}{\sqrt{N_{0} A^{(M)}}}\right)\right)^{M}$. For the case of the slot containing the signal pulse, the cumulative distribution function of the maximum output-denoted by $Y_{\max }^{(s)}$-is

$$
F(y)=\prod_{m=1}^{M} Q\left(-\frac{\left(y-\Lambda_{m}\right)}{\sqrt{N_{0} A^{(M)}}}\right)
$$


For large $M, \Lambda_{m} \approx \mathcal{S}_{m} A^{(M)}$ where $\mathcal{S}_{m}=\frac{\lambda_{p}}{2 \pi \rho^{2}} \exp \left(-\frac{\left(x_{m}-x_{0}\right)^{2}+\left(y_{m}-y_{0}\right)^{2}}{2 \rho^{2}}\right)$, where $\left(x_{m}, y_{m}\right)$ denotes the center of the $m$ th detector. Therefore, for large $M$,

$$
F(y) \approx \prod_{m=1}^{M} Q\left(-\frac{\left(y-\mathcal{S}_{m} A^{(M)}\right)}{\sqrt{N_{0} A^{(M)}}}\right)=\prod_{m=1}^{M} Q\left(-\frac{y}{\sqrt{N_{0} A^{(M)}}}+\frac{\mathcal{S}_{m} \sqrt{A^{(M)}}}{\sqrt{N_{0}}}\right)
$$

and since $A^{(M)} \approx 0$ for large $M$,

$$
F(y) \approx \prod_{m=1}^{M} Q\left(-\frac{y}{\sqrt{N_{0} A^{(M)}}}\right)=\left(Q\left(-\frac{y}{\sqrt{N_{0} A^{(M)}}}\right)\right)^{M} .
$$

Comparing (39) and (41), we note that $Y_{\max }^{(s)}$ and $Y_{\max }^{(n)}$ are identically distributed and independent for large $M$. In this case, $P\left(\left\{Y_{\max }^{(s)}>Y_{\max }^{(n)}\right\}\right)=\frac{1}{2}$ which can be shown by the following argument. For two identically distributed random variables $X$ and $Y, F_{X}(z)=F_{Y}(z)=F(z)$, where $F_{X}(\cdot)$ and $F_{Y}(\cdot)$ are cumulative distribution functions of $X$ and $Y$, respectively. Then, the probability that $Y<X$ is,

$$
\begin{aligned}
\mathbb{P}(\{Y<X\}) & =\int_{-\infty}^{\infty} \mathbb{P}(\{Y<x\}) d F(x)=\int_{-\infty}^{\infty} F(x) d F(x) \\
& =\int_{0}^{1} u d u=\frac{1}{2} .
\end{aligned}
$$

Therefore, for large $M$, the probability of error for SC (Max Output) is

$$
\lim _{M \rightarrow \infty} \mathbb{P}\left(\mathcal{E} \mid x_{0}, y_{0}\right)=1-\left(\frac{1}{2}\right)^{\mathcal{M}-1}
$$

which is the same result as the asymptotic results for SC (Max SNR) (see (28)).

\section{Switched Combiner}

The Selection Combiner (Max Output) sorts through the detector outputs every symbol time in order to choose the detector that has the maximum output. This leads to a larger complexity overhead that may be reduced if the sorting procedure is carried out at a less frequent rate.

The switched combining scheme is a low complexity version of the selection combining scheme in that the search for the maximum SNR detector is not carried out every symbol time but rather at a much longer period [20]. In the switched combining scheme discussed in this paper, we begin by choosing the output of the detector with the maximum SNR as our sufficient statistic for making a decision on a symbol. However, for the next group of symbols, we use the output of the same detector for symbol decisions unless the SNR of the said detector goes below a certain predefined threshold. In this sense, 
we save ourselves the sorting complexity every symbol time that we run into with a selection combining scheme. However, since we do not necessarily use the output of the maximum SNR detector in this case in order to make decisions, the error probability of the switched combiner will be strictly larger than the error probability of the selection combiner.

Before we begin on the definition of the switched combiner, let us first explain a few preliminaries. Let us assume that the beam position is a random process (specifically, a Gauss-Markov Process), and we denote this random process by $\mathcal{X}[n] \triangleq\left[X_{0}[n] \quad Y_{0}[n]\right]^{T}$, where $\left(x_{0}[n], y_{0}[n]\right)$ is a realization of $\left(X_{0}[n], Y_{0}[n]\right)$. We further assume that we can perfectly track $\left(X_{0}[n], Y_{0}[n]\right)$ on the array.

The beam center evolves in time as a Gauss-Markov process by the following rule:

$$
\mathcal{X}[n]=\boldsymbol{\Phi} \mathcal{X}[n-1]+\boldsymbol{V}[n]
$$

where $\boldsymbol{V}[n]$ is a zero-mean $2 \times 1$ vector that represents a white noise process. Specifically, $\boldsymbol{V}[n]=$ $\left[V_{x}[n] \quad V_{y}[n]\right]^{T}$, where $V_{x}[n] \perp V_{y}[m]$ for any discrete-time indices $n$ and $m$. Additionally, we assume that $V_{x}[n] \perp V_{x}[m]$ and $V_{y}[n] \perp V_{y}[m]$ whenever $n \neq m$. Moreover, for the sake of simplicity, we assume that $\mathbb{E}\left[V_{x}^{2}[n]\right]=\mathbb{E}\left[V_{y}^{2}[n]\right]=\sigma_{V}^{2}$.

For the purpose of computing the probability of error for a switched combiner, let us assume that the beam tracking system estimates the beam position every $\ell_{s}$ symbols, and adjusts the beam position to the center of the array every $\ell_{a}$ symbols. Let us assume that $\ell_{a}=k_{0} \ell_{s}$ for some positive integer $k_{0}$. This means that the tracking algorithm "senses" or estimates the beam position every $\ell_{s}$ time units and "aligns" or adjusts the beam center to the center of array every $\ell_{a}$ time units. We call $\ell_{s}$ the beam position sensing period, and $\ell_{a}$ the beam position alignment period. We assume that the tracking system performs perfect tracking and that the beam center and the center of the array are aligned perfectly (and instantly) every $\ell_{a}$ time units. We thus have that

$$
\begin{aligned}
& \sigma_{R}^{2} \triangleq \ell_{a} \sigma_{V}^{2} \\
& \sigma_{R_{0}}^{2} \triangleq \ell_{s} \sigma_{V}^{2}=\ell_{s} \frac{\sigma_{R}^{2}}{\ell_{a}}=\frac{\sigma_{R}^{2}}{k_{0}}
\end{aligned}
$$

where $\sigma_{R_{0}}$ is the scale parameter of the beam center $\left(x_{0}, y_{0}\right)$ every sensing period, and $\sigma_{R}$ is the scale parameter every alignment period.

It is not straightforward to compute an analytical expression for the probability of error for a switched combiner. Hence, we resort to the following Monte Carlo simulations based method for computing the 
average probability of error for the switched combining scheme.

\section{1) Switched Combiner Scheme for a Single Detector Output}

The algorithm to compute the average probability of error for our proposed switched combiner is as follows:

1) Choose $\left(x_{0}, y_{0}\right)=(0,0)$.

2) Run selection combiner, which means that we choose the detector with maximum SNR: $\Lambda^{(0)} \triangleq$ $\max \left(\Lambda_{1}, \Lambda_{2}, \ldots, \Lambda_{M}\right)$.

Note: $\Lambda_{m}$ is a function of $\left(x_{0}, y_{0}\right)$. Thus, $\Lambda_{m}=\Lambda_{m}\left(x_{0}, y_{0}\right) \Longrightarrow \Lambda^{(0)}=\Lambda^{(0)}\left(x_{0}, y_{0}\right)$.

3) Compute probability of error $\mathbb{P}(\mathcal{E})$ for the chosen detector:

$$
\mathbb{P}(\mathcal{C})=\left(P\left(\left\{Y_{s}-Y_{n}>0\right\}\right)\right)^{\mathcal{M}-1}
$$

where $Y_{s} \sim \mathcal{N}\left(\Lambda^{(0)}, N_{0} A^{(M)}\right)$, and $Y_{n} \sim \mathcal{N}\left(0, N_{0} A^{(M)}\right)$. Then,

$$
\mathbb{P}(\mathcal{E})=1-\left(Q\left(-\frac{\Lambda^{(0)}}{\sqrt{2 N_{0} A^{(M)}}}\right)\right)^{\mathcal{M}-1} .
$$

4) Choose a new $\left(\Delta x_{0}, \Delta y_{0}\right) \sim \mathcal{N}\left(\mathbf{0}, \boldsymbol{\Sigma}_{R_{0}}\right)$, where $\boldsymbol{\Sigma}_{R_{0}} \triangleq\left[\begin{array}{cc}\sigma_{R_{0}}^{2} & 0 \\ 0 & \sigma_{R_{0}}^{2}\end{array}\right]$ and update the beam position as

$$
\begin{aligned}
& x_{0}=x_{0}+\Delta x_{0} \\
& y_{0}=y_{0}+\Delta y_{0} .
\end{aligned}
$$

5) If $\frac{\Lambda^{(0)}\left(x_{0}, y_{0}\right)}{N_{0} A}<\gamma$, then go to Step 2 and choose a new detector with maximum SNR and update $\Lambda_{0}$. Else, go to Step 3.

6) Repeat until $k_{0}$ values of $\mathbb{P}(\mathcal{E})$ are obtained.

The average probability of error is obtained by averaging over all the probabilities of error obtained in the simulation.

Finally, we consider an important point regarding the threshold $\gamma$. Let us define

$$
\Lambda^{*} \triangleq \int_{-\ell(A) / 2}^{\ell(A) / 2} \int_{-\ell(A) / 2}^{\ell(A) / 2} \frac{\lambda_{p}}{2 \pi \rho^{2}} \exp \left(-\frac{x^{2}+y^{2}}{2 \rho^{2}}\right) d y d x
$$

where $\ell(A)$ is the length any one side of the square detector in the array. The quantity $\Lambda^{*}$ is the maximum 
possible signal power that can be obtained in any detector of the array at any time. Therefore, for any $\gamma \geq \frac{\Lambda^{*}}{N_{0} A^{(M)}}$, the SWC and the SC are essentially the same algorithm.

\section{Simulation Results and Commentary}

\begin{tabular}{|c|c|c|}
\hline Notation(s) & Name / Unit & Default Values \\
\hline$\lambda_{p}$ & Peak power (Watt) & 1 \\
\hline$N_{0}$ & Noise power (Watts $\left./ \mathrm{mm}^{2}\right)$ & 0.06 \\
\hline$|\mathcal{A}|$ & Area of array detector $\left(\mathrm{mm}^{2}\right)$ & 4 \\
\hline$\rho$ & Beam radius $(\mathrm{mm})$ & 0.2 \\
\hline$a$ & Area of single detector $\left(\mathrm{mm}^{2}\right)$ & 0.05 \\
\hline$\sigma_{R}^{2}$ & Jitter variance $(\mathrm{mm})$ & 0.05 \\
\hline$\left(x_{0}, y_{0}\right)$ & Beam location $(\mathrm{mm})$ & $(0,0)$ \\
\hline$M$ & Number of Detectors & 16 \\
\hline $\mathcal{M}$ & PPM modulation order & 8 \\
\hline
\end{tabular}

Fig. 7. A table of default value(s) and its range for the experiment in the paper

We now illustrate the simulations results that we have obtained in this study. In these simulations, we have considered the Gaussian channel. For all the experiments, the area of the detector array $|\mathcal{A}|=4 \mathrm{~mm}^{2}$. This detector array stretches from $-1 \mathrm{~mm}$ to $1 \mathrm{~mm}$ in each of the two dimensions, and the center of the array coincides with the origin. Additionally, we want to highlight that the area of the array $|\mathcal{A}|$ is fixed irrespective of the number of detector $M$ in the array. Therefore, a larger $M$ implies a smaller area per detector. The modulation scheme in all the simulations is 8-PPM. 


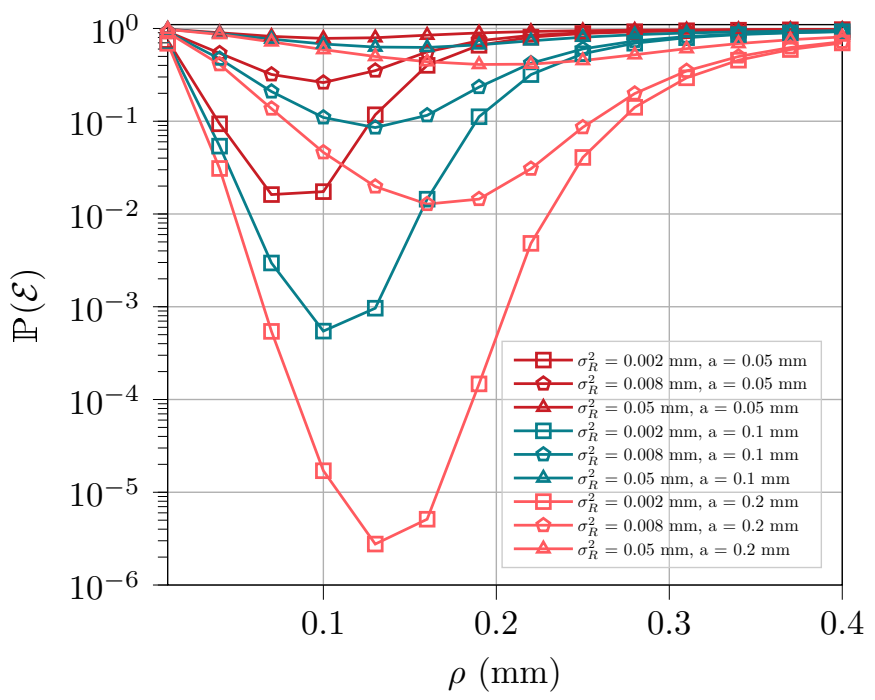

Fig. 8. The figure shows the probability of error of single detector receiver as a function of both jitter $\sigma_{R}^{2}$ and area of receiver $a$ for different values of beam radius $\rho$. The modulation scheme considered in this case is 8-PPM.

Fig. 8 shows the error probability of single detector receiver from (10) as a function of beam radius $\rho$ for different jitter and area. We note that the probability of error of the single detector is proportional to the value of jitter parameter $\sigma_{R}^{2}$ and the radius (area) of the single detector $a$.

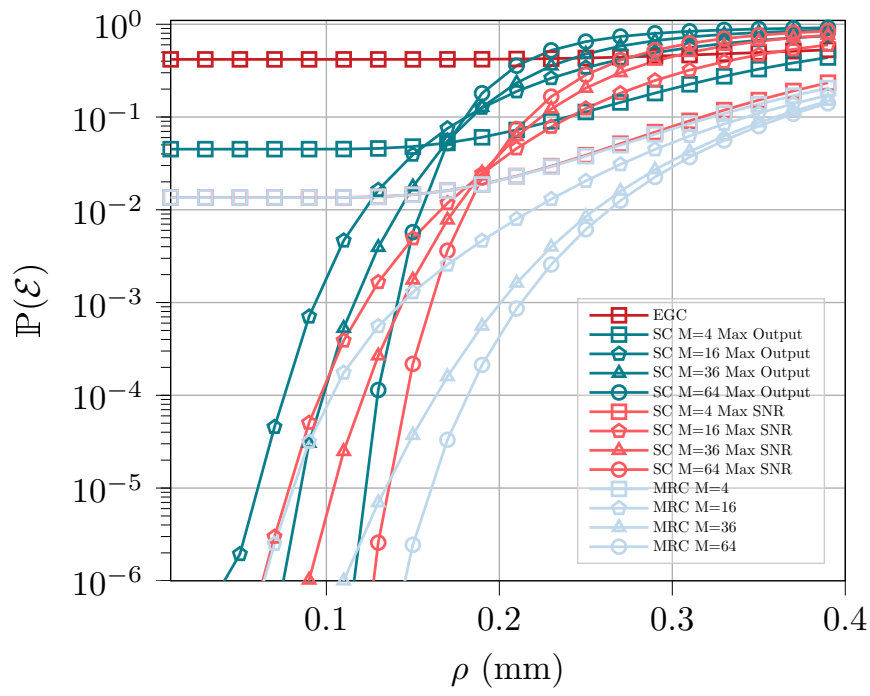

Fig. 9. The figure shows the probability of error of different combiners which are equal gain combiner (EGC), selection combiner (SC) and maximum ratio combiner (MRC) as a function of beam radius $\rho$ at beam center $(0.4,0.4)$ for the $2 \times 2,4 \times 4$ and $8 \times 8$ detector arrays. The modulation scheme considered in this case is 8-PPM. 
Fig. 9 shows the error probability of equal gain combiner (EGC) from (19), selection combiner (SC Max SNR) from (26), selection combiner (SC Max Output) from (37), and maximum ratio combiner (MRC) from (20) for the detector array receiver, as a function of beam radius $\rho$ for $2 \times 2,4 \times 4$ and $8 \times 8$ array configurations for a fixed beam center position: $\left(x_{0}, y_{0}\right)=(0.4,0.4)$. We observe that the MRC obtains the best error probability performance followed by SC (Max SNR), SC (Max Output) and EGC. This is straightforward since MRC weights the output of each detector optimally [11] as compared to other schemes that weight the detector outputs suboptimally before the fusion takes place.

Fig. 10 considers the probability of error for the combining schemes except that beam center $\left(x_{0}, y_{0}\right)$ is now modeled as a random variable. The distribution of $\left(x_{0}, y_{0}\right)$ is $(\mathcal{N}(\mathbf{0}, \boldsymbol{C}))$ where $\mathbf{0}$ is the twodimensional column vector of zeros, and $\boldsymbol{C} \triangleq\left[\begin{array}{cc}\sigma_{R}^{2} & 0 \\ 0 & \sigma_{R}^{2}\end{array}\right]$. Here, we see the same trend: The MRC provides the best probability of error performance, followed by SC (Max SNR), SC (Max Output) and the EGC.

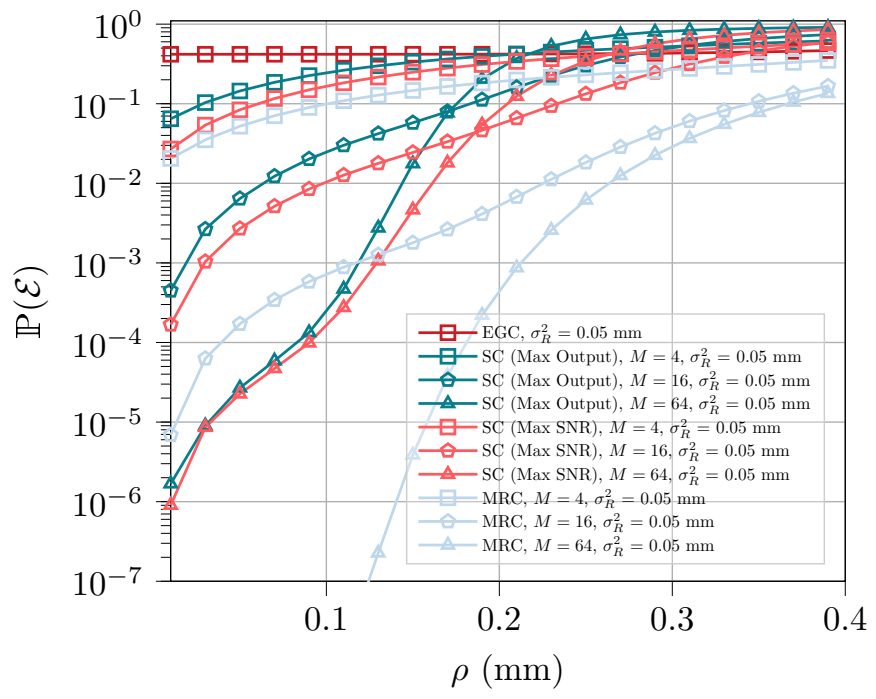

Fig. 10. This figure shows the effect of beam radius on the average error probability with jitter $\sigma_{R}^{2}=0.05 \mathrm{~mm}$ for the $2 \times 2,4 \times 4$ and $8 \times 8$ EGC, SC and the MRC combining schemes. The value of noise parameter $N_{0}$ is $0.06 \mathrm{Watt} / \mathrm{mm}^{2}$. 


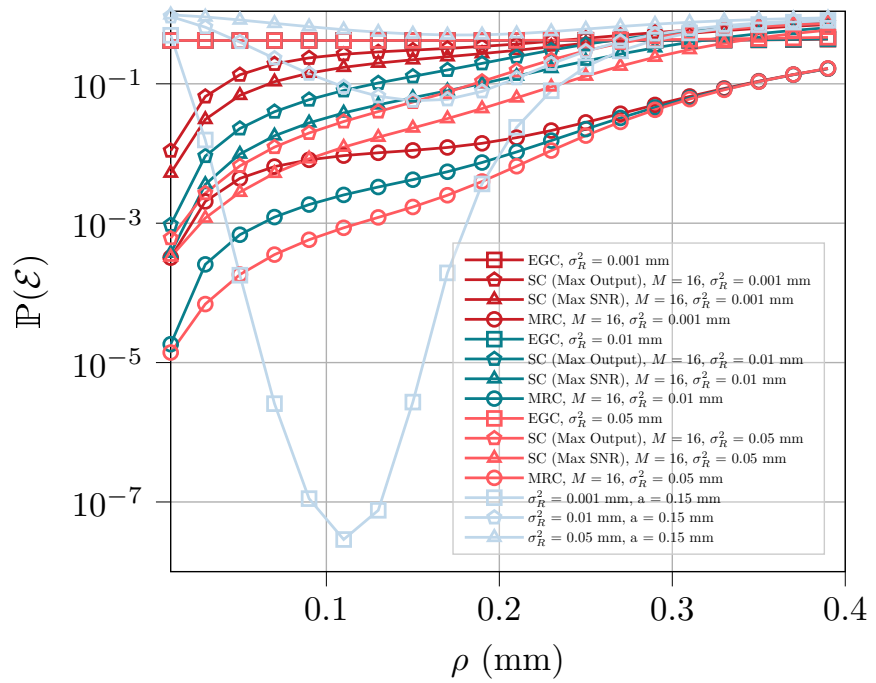

Fig. 11. This figure shows the effect of beam radius on the average error probability. The performance of the single detector with radius $a=0.05 \mathrm{~mm}$ is compared with the $4 \times 4 \mathrm{EGC}$, SC and MRC combining schemes. The jitter variance $\sigma_{R}^{2}=0.05 \mathrm{~mm}$. The value of noise parameter $N_{0}$ is $0.06 \mathrm{Watt} / \mathrm{mm}^{2}$.

Fig. 11 shows the comparison of single detector and different array detectors as a function of $\rho$ for jitter variance $\sigma_{R}^{2}$ in the range $0.001-0.05 \mathrm{~mm}$. The result indicates that the single detector within a certain range of beam radius $\rho$ has the ability to outperform the detector array combining schemes, especially for small jitter variance $\sigma_{R}^{2}$. For the single detector, the beam radius $\rho$ is indeed a parameter that can be optimized in order to obtain a minimum probability of error.

Regarding the array of detectors, we note that the error probability performance degrades for a small jitter variance $\sigma_{R}^{2}$ for the MRC and SC schemes of the $4 \times 4$ array. This is explained (nonrigorously) as follows: For a smaller jitter variance, the beam center $\left(x_{0}, y_{0}\right)$ is practically localized at the point $(0,0)$, which is the point at which the corners of the four inner detectors of the $4 \times 4$ array meet or conjoin. When $\left(x_{0}, y_{0}\right)$ is localized to $(0,0)$, then most of the energy in the beam is distributed uniformly in these four detectors, and no single detector has a significantly better SNR than other detectors. However, in the scenario when $\left(x_{0}, y_{0}\right)$ coincides with the center of a given detector, a big chunk of energy will be localized within the said detector which leads to a very high SNR in that detector. Since, the MRC weights the output of each detector in proportion to its SNR, the detectors with higher SNR get substantially higher weights than detectors with low SNR [9]. This leads to an overall increase in the SNR in the final sufficient statistic which leads to a smaller probability of error. This roughly explains why the MRC and SC schemes 
do not perform so well for the $4 \times 4$ array for small jitter variance (when the beam center resides close to the center of the array).

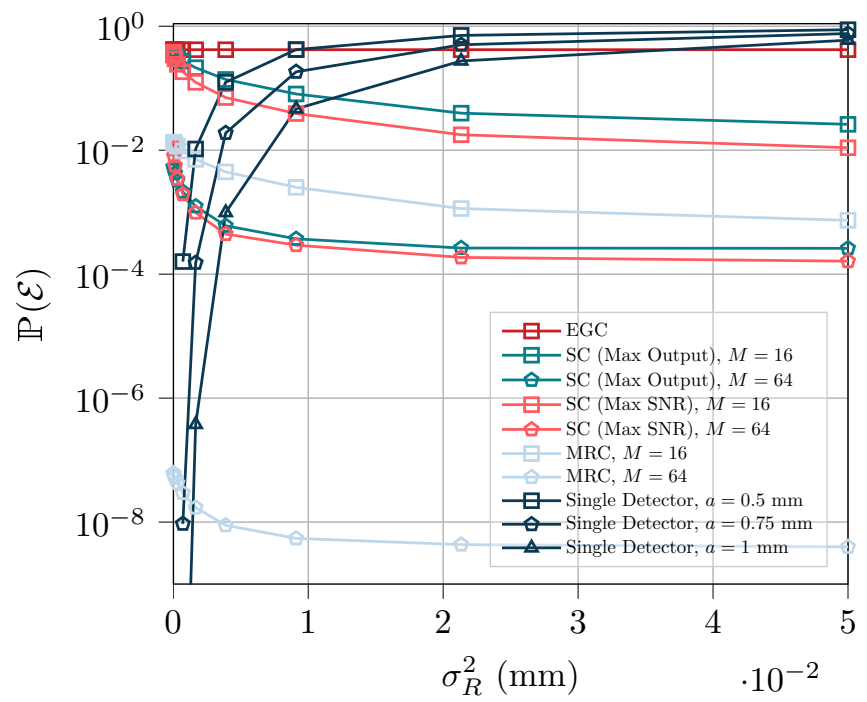

Fig. 12. The figure shows the comparison of average error probability between the single detector and the array of detectors as a function of jitter variance $\sigma_{R}^{2}$. The value of noise parameter $N_{0}$ is $0.06 \mathrm{Watt} / \mathrm{mm}^{2}$. The optimal beam radius is chosen for single detector in this case. For the array of detectors, the minimum possible radius is chosen $(\rho=0.1 \mathrm{~mm})$.

Fig. 12 shows the comparison of single detector for different detector size $a$ and different array detectors as a function of jitter $\sigma_{R}^{2}$ for $4 \times 4$ and $8 \times 8$ array configuration. In this figure, we are using the optimal beam radius for the single detector, and the smallest possible beam radius for the array of detectors (whic is $0.1 \mathrm{~mm}$ ). The result indicates single detector with low jitter has ability to outperform some array combining schemes. Besides that, the single detector probability of error experiences a performance gain in proportion to the detector radius $a$. 


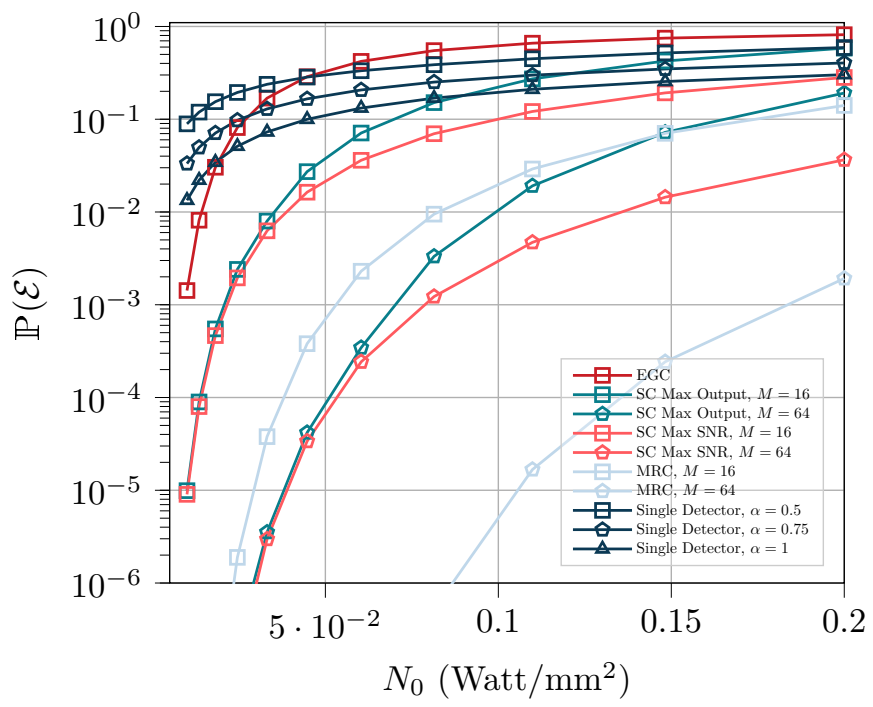

Fig. 13. The figure shows the comparison of average error probability between the single detector with different power spilt $\alpha$ from 0.5 to 1 and the $4 \times 4$ and $8 \times 8$ combiner (EGC), selection combiner(SC) and maximum ratio combiner(MRC) detector arrays as function of noise $N_{0}$. The value of noise parameter $\sigma_{R}^{2}$ is $0.05 \mathrm{~mm}$ and the beam location is $(0.4,0.4)$.

Fig. 13 shows the performance comparison between the single detector and the array of detectors as a function of noise parameter $N_{0}$ when $\left(x_{0}, y_{0}\right)$ is fixed at $(0.4,0.4)$. The comparison is made for different power split factors $\alpha$ for the single detector receiver ${ }^{3}$. As expected, the single detector performance increases in proportion to power split factor $\alpha$.

${ }^{3}$ Here, we want to emphasize that even though the case of $\alpha=1$ is also shown in Fig. 13, $\alpha$ is strictly less than 1 in a practical FSO receiver. 


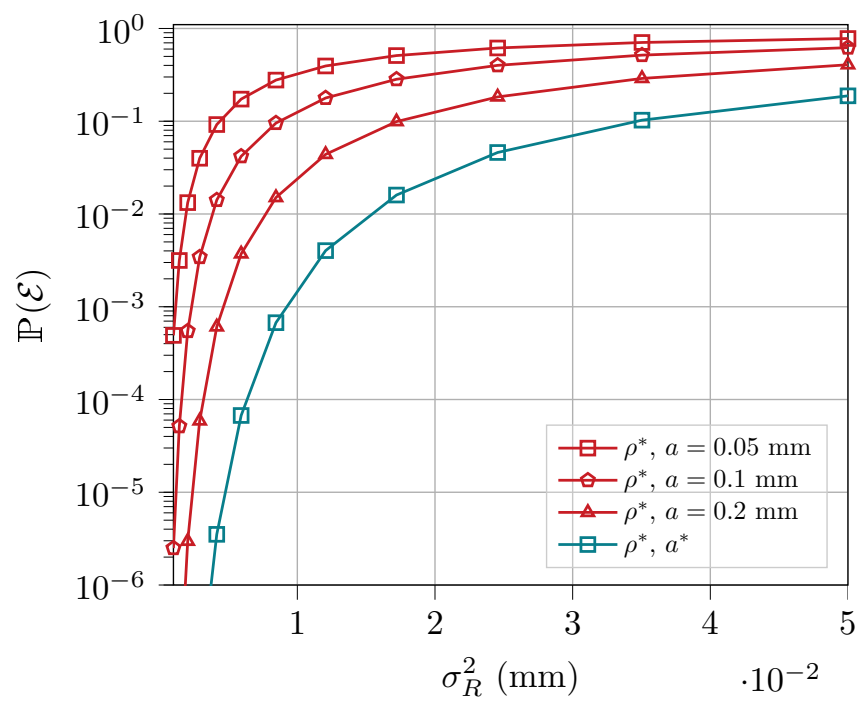

Fig. 14. The figure shows the error probability of single detector as a function of jitter variance $\sigma_{R}^{2}$ for different values of detector radius $a$ and the corresponding optimized beam radius $\rho^{*}$. The value of noise parameter $N_{0}$ is $0.06 \mathrm{Watt} / \mathrm{mm}^{2}$. The optimum value $\rho *$ is found over the range $0.1<\rho<0.4 \mathrm{~mm}$, and the optimum value $a^{*}$ is obtained over the range $0.05<a<0.4 \mathrm{~mm}$.

Fig. 14 shows the comparison of optimized beam radius $\rho$ of single detector for different radii $a$ for the single detector case as a function of jitter variance $\sigma_{R}^{2}$. We note that we obtain a better probability of error performance if the optimization is carried out over two dimensions (both $a$ and $\rho$ ) as opposed to just one dimension (only $\rho$ ). 


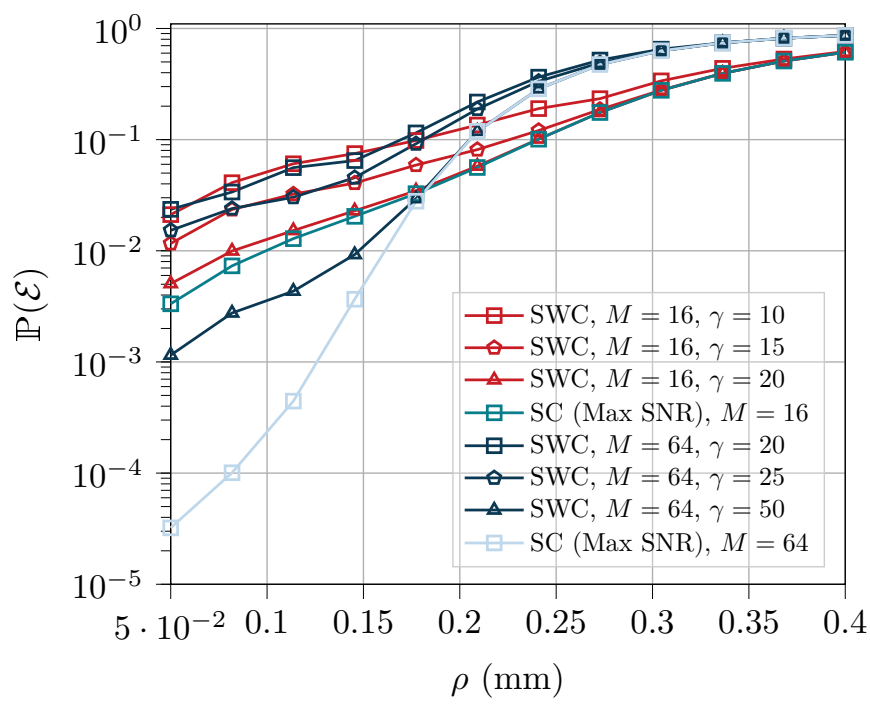

Fig. 15. The figure shows the error probability of $4 \times 4$ and $8 \times 8$ switched combiner (SWC) for different threshold $\gamma$ and selection combiner (SC) as a function of beam radius $\rho$. The value of noise parameter $N_{0}$ is $0.06 \mathrm{Watt} / \mathrm{mm}^{2}$.

Fig. 15 illustrates the performance comparison between the SC (Max SNR) and the switched combiner as a function of beam radius $\rho$ for different switching threshold $\gamma$. For the SWC, we assumed that the data rate on the optical channel is $10^{7} 8$-PPM symbols every second. We additionally assumed that the beam position sensing period $\ell_{s}=10$ symbol time, and the beam alignment update period $\ell_{a}=10^{4}$ symbol time. This implies that $k_{0}=1000$. The period $\ell_{a}=10^{4}$ means that the beam is aligned to the center of the array every 1 milliseconds.

For SWC, a larger threshold $\gamma$ implies a more frequent switching to the optimum (largest SNR) detector, which means that the performance of SWC will be very close to the performance of SC (Max SNR). However, a larger threshold $\gamma$ shall also imply a larger computational complexity. 


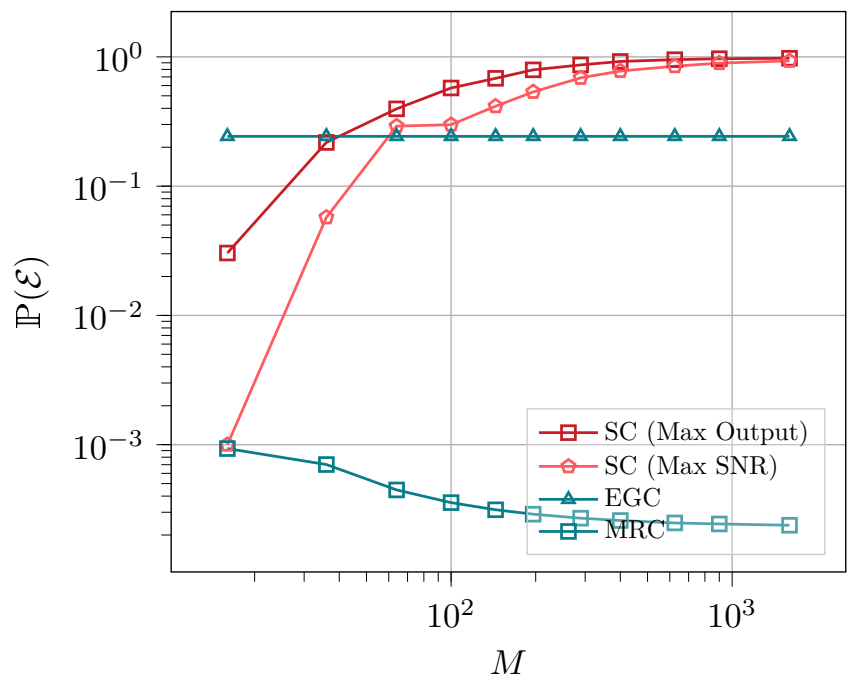

Fig. 16. This figure shows the probability of error as a function of number of detectors $M$ in the array. The values of parameters are as follows: noise parameter $N_{0}=0.04$ Watts $/ \mathrm{mm}^{2}$, beam radius $\rho=0.25 \mathrm{~mm}$, and the beam location $\left(x_{0}, y_{0}\right)=(0.25,0.25)$. The area of the array $\mathcal{A}=4 \mathrm{~mm}^{2}$.

Fig. 16 shows the probability of error obtained with an array of detectors for the limiting case of $M \rightarrow \infty$ when the array area is held constant at $\mathcal{A}=4 \mathrm{~mm}^{2}$. We note that the error probability of MRC decays monotonically with $M$. Meanwhile, the selection combiner SC (Max SNR) and SC (Max Output) suffer an increase in the probability of error for large $M$ - the probabilities of error for both types converge to $1-\left(\frac{1}{2}\right)^{\mathcal{M}-1}$ from (28) and (43). The EGC shows no difference in performance regardless of the number of detectors $M$.

\section{Complexity Analysis}

In this section, we briefly analyze the complexity for different combining schemes and compare it with the complexity of a single-detector receiver. Since the EGC simply adds the outputs of all the array elements to form the sufficient statistic [11], it requires $M$ additions. Therefore, its complexity is $\mathcal{O}(M)$. Regarding the SC, both the Max SNR and Max output versions require a sorting algorithm to find the maximum of the $M$ output values. Therefore, selection combiner has a complexity of $\mathcal{O}(M \log (M))$ if we use merge sorting. For the switching combiner (SWC), the sorting complexity is $\beta \mathcal{O}(M \log (M))$ where $0<\beta<1$ and $\beta$ depends on the threshold $\gamma$. For the MRC, we have $M$ multiplications and $M$ additions [11]. Hence, its complexity is $\mathcal{O}\left(M^{2}\right)$. 
The SC (Max SNR) and MRC incur additional complexity since they require the estimation of beam parameters - such as peak intensity $\lambda_{p}$, the beam radius $\rho$ and the location of the beam center $\left(x_{0}, y_{0}\right)$ on the array-in order to form the sufficient statistic. The value of beam radius $\rho$ in the focal plane depends on the lensing arrangement; hence it is treated as a known deterministic quantity. The estimation of $\lambda_{p}$ is straightforward via a method of moments estimator [21], and we may ignore its complexity. Regarding the estimation of $\left(x_{0}, y_{0}\right)$, if we use a centroid estimator, we need a total of $3 M$ additions and $2 M$ multiplications (please see the definition of the centroid estimator in [2] or [22]). Thus, this extra complexity has to be incorporated into the total complexity of MRC, SC (Max SNR) and SWC schemes.

Additionally, we want to point out that there is some hardware complexity associated with an array of detectors as well. For instance, if the number of detectors is very large, the optical fill factor might be too small. Thus, in this scenario, we may have to place a microlens array in front of the array of detectors in order to focus the energy away from the dead area into the active area (the detectors). This will minimize the effect of a reduced optical fill factor.

Regarding the single-detector receiver, the total computational complexity is the complexity required in the tracking assembly which-if a centroid estimator is used-turns out to be $3 M^{\prime}$ additions and $2 M^{\prime}$ multiplications, where $M^{\prime}$ is the number of elements used in the detector array in the tracking assembly. Hence, the complexity of a single-detector receiver is $\mathcal{O}\left(M^{\prime}\right)$. In addition to this, there is a complexity associated with the single-detector receiver concerning the optimization of the beam radius that depends on the jitter variance $\sigma_{R}^{2}$. However, if $\sigma_{R}^{2}$ remains almost stationary over the course of say, a few million symbols (which takes less than a second for most short-to-medium distance FSO links), the complexity associated with optimization of beam radius may be ignored relative to the complexity incurred every symbol time.

Fig. 17 summarizes the complexities of different combining schemes for an array of detectors and a single detector. 


\begin{tabular}{|l|l|}
\hline Scheme & Complexity \\
\hline EGC & $\mathcal{O}(M)$ \\
\hline SC (Max Output) & $\mathcal{O}(M \log (M))$ \\
\hline SC (Max SNR) & $\mathcal{O}(M \log (M))+\mathcal{O}(M)$ \\
\hline SWC & $\beta \mathcal{O}(M \log (M))+\mathcal{O}(M)$ \\
\hline MRC & $\mathcal{O}\left(M^{2}\right)+\mathcal{O}(M)$ \\
\hline Single-Detector & $\mathcal{O}\left(M^{\prime}\right)$ \\
\hline
\end{tabular}

Fig. 17. A table summarizing the computational complexities of different combining schemes.

\section{Conclusion}

In this paper, we have compared the probability of error performance of a single-detector receiver with a detector array receiver in a free-space optical communication system. Concerning the array of detectors, different combining schemes were considered in the probability of error comparison. We conclude that except the equal gain combiner, all other combining schemes for the array of detectors yield a better error probability performance than the single-detector receiver for high to moderate values of jitter standard deviation. However, the array of detectors incur a higher computational overhead when compared to the single-detector receiver. Additionally, when the jitter standard deviation is very small compared to the detector radius of the single-detector receiver, the single-detector receiver outperforms the array of detectors in terms of error probability.

\section{REFERENCES}

[1] A. Trichili, M. A. Cox, B. S. Ooi, and M.-S. Alouini, "Roadmap to free space optics," J. Opt. Soc. Am. B, vol. 37, no. 11, pp. A184-A201, Nov 2020. [Online]. Available: http://josab.osa.org/abstract.cfm?URI=josab-37-11-A184

[2] M. S. Bashir and M. R. Bell, "Optical beam position estimation in free-space optical communication," IEEE Transactions on Aerospace and Electronic Systems, vol. 52, no. 6, December 2016.

[3] H. Hemmati, A. Biswas, and I. B. Djordjevic, "Deep-space optical communications: Future perspectives and applications," Proceedings of the IEEE, vol. 99, no. 11, pp. 2020-2039, 2011.

[4] O. Kharraz and D. Forsyth, "Performance comparisons between PIN and APD photodetectors for use in optical communication systems," Optik, vol. 124, no. 13, pp. 1493 - 1498, 2013. [Online]. Available: http://www.sciencedirect.com/science/article/pii/ S0030402612002859

[5] M. S. Ferraro, W. R. Clark, W. S. Rabinovich, R. Mahon, J. L. Murphy, P. G. Goetz, L. M. Thomas, H. R. Burris, C. I. Moore, W. D. Waters, K. Vaccaro, and B. D. Krejca, "InAlAs/InGaAs avalanche photodiode arrays for free space optical communication," Appl. Opt., vol. 54, no. 31, pp. F182-F188, Nov 2015. [Online]. Available: http://ao.osa.org/abstract.cfm?URI=ao-54-31-F182

[6] M. S. Bashir and M. R. Bell, "Optical beam position tracking in free-space optical communication systems," IEEE Transactions on Aerospace and Electronic Systems, vol. 20, no. 2, April 2018. 
[7] M. S. Bashir and M. R. Bell, "The impact of optical beam position estimation on the probability of error in free-space optical communications," IEEE Transactions on Aerospace and Electronic Systems, vol. 55, no. 3, pp. 1319-1333, June 2019.

[8] M. S. Bashir and S. S. Muhammad, "Time synchronization in photon-limited deep space optical communications," IEEE Transactions on Aerospace and Electronic Systems, vol. 56, no. 1, pp. 30-40, 2020.

[9] M. S. Bashir, "Free-space optical communications with detector arrays: A mathematical analysis," IEEE Transactions on Aerospace and Electronic Systems, vol. 56, no. 2, pp. 1420-1429, 2020.

[10] M. S. Bashir and M.-S. Alouini, "Signal acquisition with photon-counting detector arrays in free-space optical communications," IEEE Transactions on Wireless Communications, vol. 19, no. 4, pp. 2181-2195, 2020.

[11] M. S. Bashir and M. S. Alouini, "Free-space optical MISO communications with an array of detectors," IEEE Open Journal of the Communications Society, vol. 1, pp. 1765-1780, 2020.

[12] V. A. Vilnrotter and M. Srinivasan, "Adaptive detector arrays for optical communications receivers," IEEE Transactions on Communications, vol. 50, no. 7, pp. 1091-1097, July 2002.

[13] V. Vilnrotter, C. . Lau, M. Srinivasan, K. Andrews, and R. Mukai, "Optical array receiver for communication through atmospheric turbulence," Journal of Lightwave Technology, vol. 23, no. 4, pp. 1664-1675, April 2005.

[14] M. Srinivasan, K. S. Andrews, W. H. Farr, and A. Wong, "Photon counting detector array algorithms for deep space optical communications," in Free-Space Laser Communication and Atmospheric Propagation XXVIII, H. Hemmati and D. M. Boroson, Eds., vol. 9739, International Society for Optics and Photonics. SPIE, 2016, pp. 267 - 282. [Online]. Available: https://doi.org/10.1117/12.2217971

[15] A. A. Farid and S. Hranilovic, "Outage capacity optimization for free-space optical links with pointing errors," Journal of Lightwave Technology, vol. 25, no. 7, July 2007.

[16] V. V. Mai and H. Kim, "Adaptive beam control techniques for airborne free-space optical communication systems," Applied Optics, vol. 57, no. 26, September 2018.

[17] M. -S. Alouini and M. K. Simon, "Performance of generalized selection combining over Weibull fading channels," in IEEE 54th Vehicular Technology Conference. VTC Fall 2001. Proceedings (Cat. No.01CH37211), vol. 3, 2001, pp. 1735-1739 vol.3.

[18] M. K. Simon and M. -S. Alouini, "Performance analysis of generalized selection combining with threshold test per branch (T-GSC)," in GLOBECOM'01. IEEE Global Telecommunications Conference (Cat. No.01CH37270), vol. 2, 2001, pp. 1176-1181 vol.2.

[19] Hong-Chuan Yang and M. -S. Alouini, "MRC and GSC diversity combining with an output threshold," IEEE Transactions on Vehicular Technology, vol. 54, no. 3, pp. 1081-1090, 2005.

[20] Hong-Chuan Yang and M. . Alouini, "Performance analysis of multibranch switched diversity systems," IEEE Transactions on Communications, vol. 51, no. 5, pp. 782-794, 2003.

[21] M. S. Bashir, M.-C. Tsai, and M.-S. Alouini, "Robust beam position estimation with photon counting detector arrays in free-space optical communications," Journal of Lightwave Technology, November 2020, submitted for publication (available on engrXiv at https: //engrxiv.org/nqf3y/).

[22] D. L. Snyder and M. I. Miller, Random Point Processes in Time and Space. New York, NY: Springer-Verlag, 1991. 